\title{
IMPROVED CHARGE COLLECTION OF THE BURIED P-I-N A-Si:H RADIATION DETECTORS
}

\author{
I. Fujieda, G. Cho, M. Conti, J. Drewery, S.N. Kaplan, V. Perez-Mendez, S. Qureshi \\ Lawrence Berkeley Laboratory, Berkeley, CA.94720. \\ R.A. Street \\ Xerox Palo Alto Research Center, Palo Alto CA.94304.
}

\section{Abstract}

Charge collection in hydrogenated amorphous silicon (a$\mathrm{Si}: \mathrm{H})$ radiation detectors is improved for high LET particle detection by adding thin intrinsic layers to the usual $p-i-n$ structure. This buried $p-i-n$ structure enables us to apply higher bias and the electric field is enhanced. When irradiated by $5.8 \mathrm{MeV} \alpha$ particles, the $5.7 \mu \mathrm{m}$ thick buried $\mathrm{p}$-i-n detector with bias $300 \mathrm{~V}$ gives a signal size of 60,000 electrons, compared to about 20,000 electrons with the simple p-i-n detectors. The improved charge collection in the new structure is discussed. The capability of tailoring the field profile by doping a-Si:H opens a way to some interesting device structures.

\section{INTRODUCTION}

Hydrogenated amorphous silicon (a-Si:H) has been investigated as a possible alternative for radiation detector material for high energy physics experiments, medical imaging, material and life science studies $[1,2,3,4]$. Since a$\mathrm{Si}: \mathrm{H}$ is prepared by decomposition of $\mathrm{SiH}_{4}$ gas in glow discharge on low temperature substrates, large area detectors can be easily fabricated at low cost. The disordered Si-Si network makes the material less sensitive to radiation-induced damages, which can be annealcd at low temperature subsequently. Doping is done by mixing a boron or phosphorus containing gas such as $\mathrm{B}_{2} \mathrm{H}_{6}$ or $\mathrm{PH}_{3}$ to the $\mathrm{SiH}_{4}$ during the glow discharge deposition: under these condition, abrupt composition changes are possible. Although its material parameters such as carrier mobility, life time and defect density are less attractive than those of crystalline silicon, there are various applications in which the abovementioned advantages of a-Si: $\mathrm{H}$ outweigh its less attractive material parameters.

A-Si:H radiation detectors with simple structures such as Schottky or $\mathrm{p}-\mathrm{i}-\mathrm{n}$ diodes have been fabricated and tested with charged particles, $\mathrm{X}$-rays and $\gamma$ rays. Our efforts have been focused on making thick a-Si:H layers to increase the energy

This work was supported by the Director, Office of Energy Research, Office of High Energy and Nuclear Physics, Division of High Energy Physics, and Office of Health and Environmental Research, Division of Physics and Technological Research of the U.S. Department of Energy under contract No. DE-AC03-76SF00098. deposition by incoming radiation in the material, while maintaining the carrier transport properties. It has been shown that electrict field can extend in thick a-Si:H layers up to 40 $\mu \mathrm{m}$ [1]. The highest reverse-bias applicable to these diodes is limited by the onset of the low-frequency "pop-com" type noise[ 1,2$]$. This highest bias per unit length of a-Si:H layer ranges from about 5 to $20 \mathrm{~V} / \mu \mathrm{m}$. Therefore, $50 \mu \mathrm{m}$ layers with the present-quality a-Si:H cannot be fully-depleted unless this noise is suppressed. Also, wide variation in this highest bias is observed even among samples made from a single deposition run.

We have recently fabricated a novel type of a-Si:H radiation detector which enables us to apply higher bias before the onset of the popcom noise. The device structure and its properties are described in the next section.

\section{BREAKDOWN AND BURIED P-I-N STRUCTURE}

The "pop-com" noise is possibly due to the micro-plasma breakdown in the vicinity of the diode surface or the $p / i$ interface where the field strength becomes maximum. Any material inhomogenity close to the surface creates very high local fields and avalanche multiplication of carriers takes place. The high carrier density caused by the avalanche quenches the high local field and the avalanche diminishes. If this hypothesis of the micro-plasma breakdown in the vicinity of the surface is correct, the "pop-com" noise can be suppressed by either improving the material homogeneity or displacing the maximum field region away from the surface. We have decided to take the latter approach and developed a new structure. Intrinsic layers and lightly-doped layers were successively deposited to make a structure consisting of glass substrate/Cr/0.03- $\mathrm{n}^{+} / 0.3-\mathrm{i} / 0.03-\mathrm{n} / 5-\mathrm{i} / 0.03-\mathrm{p} / 0.3-\mathrm{i} / 0.03-\mathrm{p}^{+} / \mathrm{Cr}$. Numbers are thickness of each layer in $\mu \mathrm{m}$; the top $\mathrm{Cr}$ electrode is a disk of $3 \mathrm{~mm}$ in diameter. The dopant gas concentrations for the lightly-doped layers are determined from the preparation conditions to give a proper dopant density[5]. The fixed charge-center densities in the lighly-doped layers and the intrinsic layers under bias are estimated to be $3 \times 10^{17} \mathrm{~cm}^{-3}$ and $6-8 \times 10^{14} \mathrm{~cm}^{-3}$, respectively. The electric field profile is obtained by solving the Poisson equation in each layer and connecting the solutions at each layer boundary. Figure 1 shows schematically the device structures and ficld profiles of (a) the simple $\mathrm{p}$-i-n diode and (b) the buried $\mathrm{p}$-i-n diode. All

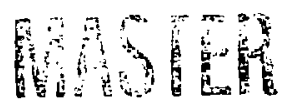


the measurements on the buried $p-i-n$ struciure reported later in this paper are obtained by these $5.7 \mu \mathrm{m}$ thick diode.

Figure 2 shows the noise characteristics of this buned $p$ - $i$ $n$ diode and a typical $p-i-n$ tiode for comparison as measured with a low noise charge-sensitive preamplifier (Tennelec TC$170)$ and a quasi-Gaussian shaping amplifier $(6.4 \mu \mathrm{sec}$ peaking time) connected to diodes. This $\mathrm{p}-\mathrm{i}-\mathrm{n}$ diode is $16 \mu \mathrm{m}$ thick and has a $3 \mathrm{~mm}$ diameter $\mathrm{Cr}$ disk top electrode. The onset of the

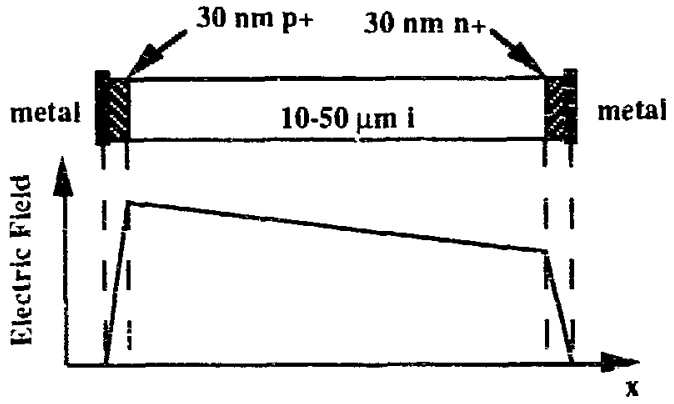

(a)

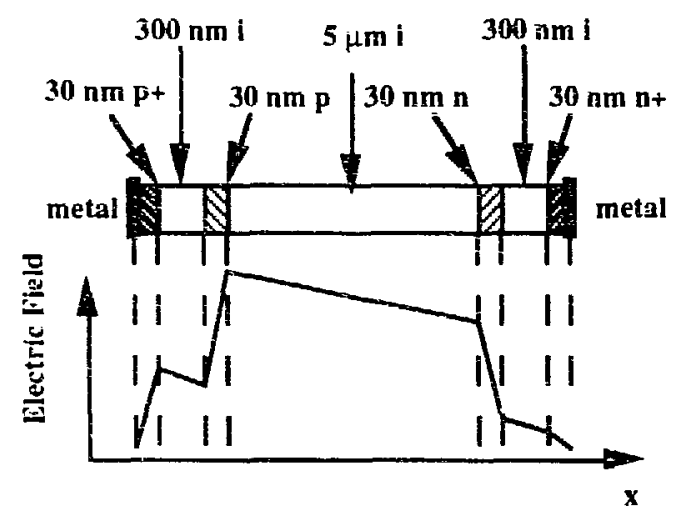

(b)

Fig.1 Schennatic drawings of the diode structure and the field profile under the depletion approximation for (a) the conventional p-i-n diode and for (b) the buried $\mathrm{p}-\mathrm{i}-\mathrm{n}$ diode.

"pop-corn" noise is around $17 \mathrm{~V} / \mathrm{\mu m}$ for the $\mathrm{p}-\mathrm{i}$-n diode. For the $5.7 \mu \mathrm{m}$ thick buried $\mathrm{p}-\mathrm{i}-\mathrm{n}$ diode with the same contact area, the initial decrease of the measured noise with bias is caused by the spread of the depletion layer. This noise level is higher than that of the $p$-i-n diode, reflecting the larger delta noise caused by its larger capacitance [6]. The increase of the noise between $100 \mathrm{~V}$ and $200 \mathrm{~V}$ is due to the slow increase of the reverse current Finally, the rapid noise increase occurs at an average field about three times larger than that in the conventional $\mathrm{p}-\mathrm{i}-\mathrm{n}$ diodes. For both cases, the sudden noise increase is not associaied with the reverse current which keeps on increasing slowly with bias even beyond the onset of the popcom noise. Its steady increase with bias is probably due to the harrier lowering by the image charge at the contacts (Schottky effect).

The onset of this breakdown varies even among samples made from a single deposition run for both the $\mathrm{p}-\mathrm{i}-\mathrm{n}$ and buricd $p^{-i-n}$ siructures. We defined the breakdown bias somewhat arbitrary as the bias at which the noise exceeds three times the minimum noise level, and conducted a systematic study of the breakdown bias. Results are shown in Fig.3. The, p-i-n diodes are all $16-17 \mu \mathrm{m}$ tinick with a $3 \mathrm{~mm}$ diameter disk $\mathrm{Cr}_{\mathrm{r}}$ top electrode made from a single deposition run. This distribution for $\mathrm{p}-\mathrm{i}-\mathrm{n}$ diodes is a representative one for different

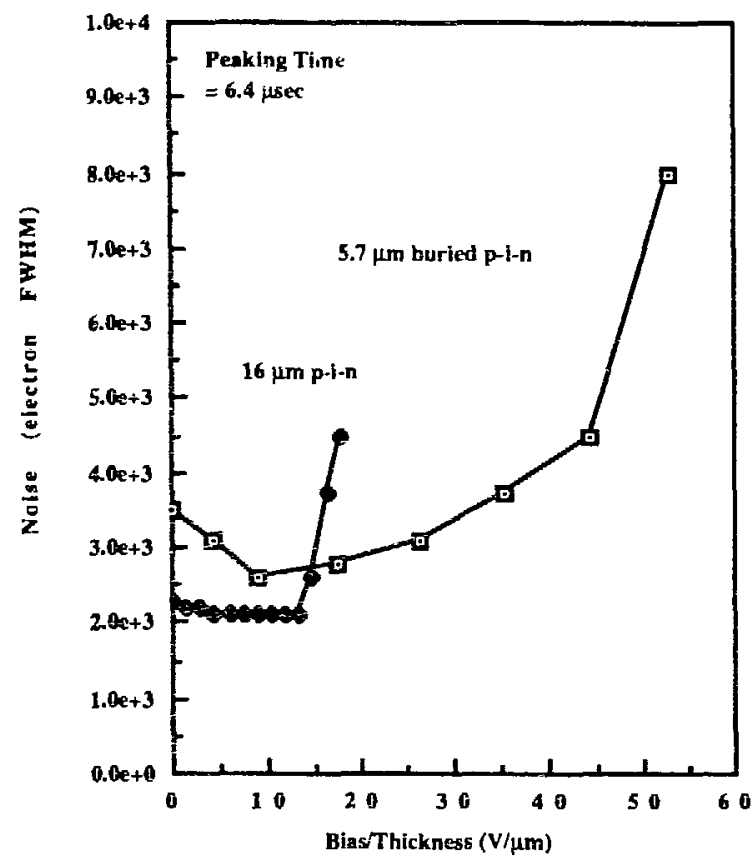

Fig. 2 Noise characteristics of the conventional $p-i-n$ diode and the buried $p-i-n$ diode. The onset of the "pop-com" noise is largely suppressed by the new structure.

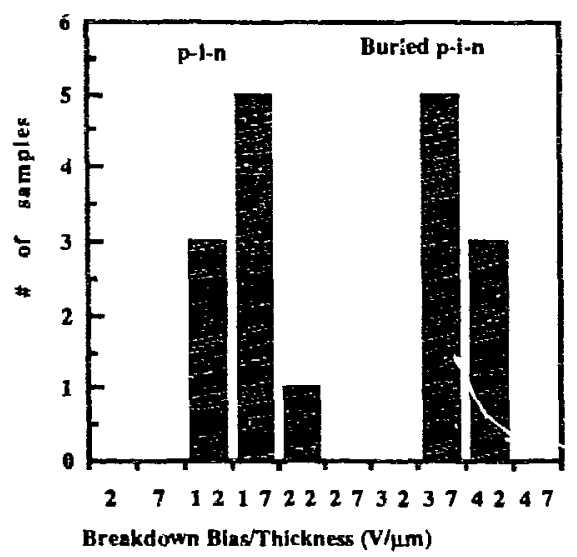

Fig.3 Variation of the breakdown bias per unit length of diodes.

inickness samples, too. The buried $p-i-n$ diodes are the ones described above. This comparison cleariy shows that the new structure is effective in suppressing the onset of the low frequency noise.

As independent evidence for the near-surface breakdown phenomenon, we conducted the following experiment. It is known in a-Si:H that defects (dangling bonds) are created 
thermally above a certain temperature where hydrogen atoms terminating unpaired Si bonds become mobile. These defects are located in the middle of the band gap and they release electrons under bias to become fixed positive space charges, which can be estimated by the time of flight (TOF) experiment

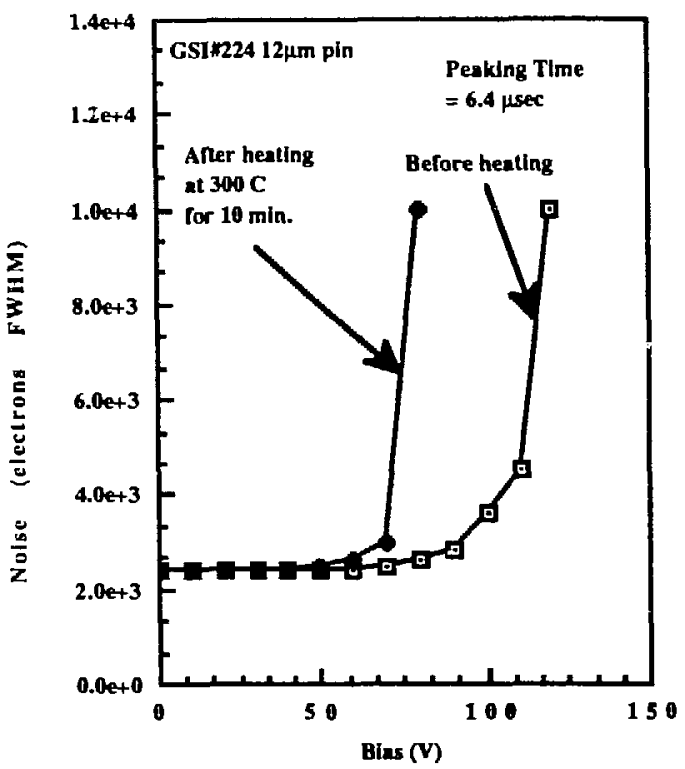

(a)

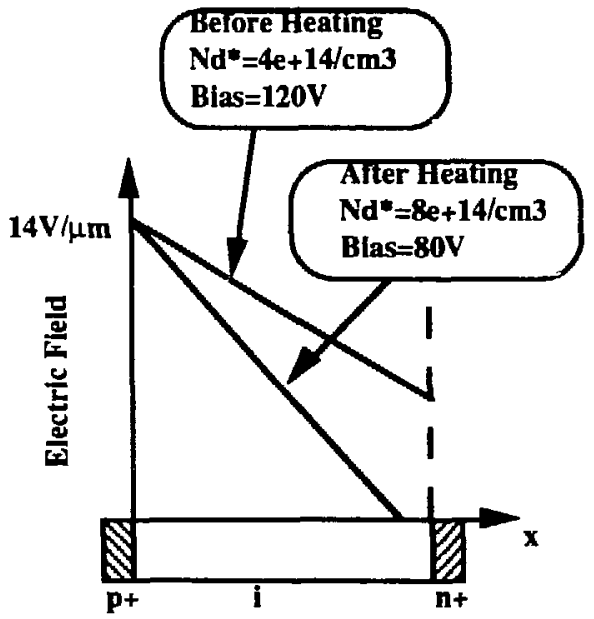

(b)

Fig.4 (a) Noise characteristics of a $12 \mu \mathrm{m}$ thick p-i-n diode before and after heating at $300^{\circ} \mathrm{C}$ for 10 minutes. (b) Field profiles at each breakdown bias before and after the heating.

[7]. This fixed charge-center density denoted as $\mathbf{N d}^{*}$ determines the field gradient under the depletion approximation. We heated a $12 \mu \mathrm{m}$ thick p-i-n diode to 300 ${ }^{\circ} \mathrm{C}$ for 10 minutes to double this defect density. The noise characteristics and $\mathrm{Nd}^{*}$ are monitored before and after the heating. Figure 4 (a) shows the noise characteristics. $\mathrm{Nd}^{*}$ was about $4 \times 10^{14} \mathrm{~cm}^{-3}$ before and $8 \times 10^{14} \mathrm{~cm}^{-3}$ after the heating. These numbers are smaller than what we have reported earlier [8] because the TOF measurements were done within 5 minutes after the bias application when the defects at the mid-gap states were still releasing electrons, although slowly. The field profiles before and after heating at each breakdown bias are calculated from each $\mathrm{Nd}^{*}$ and shown schematically in Fig.4(b). The maximum fields for both cases coincide approximately.

It has been recently reported that making the $p$ layer thicker in the $\mathrm{p}-\mathrm{i}-\mathrm{n}$ structure allows one to apply high bias on a-Si:H diodes [9]. Although the detailrd behavior of the noise and the reverse-current are not shown in [9], it is likely that a thick p layer works as a buffer layer to move the maximum field point away from the metal contact as in the case of our buried i-p layer.

\section{CHARGE COLLECTION IN THE BURIED P-I-N DIODE}

Figure 5 shows the results of pulsed light measurements. Strongly-absorbing pulsed iight ( $\lambda=510 \mathrm{~nm}, 3 \mathrm{nsec}$ wide) incident on either side of the diode simulates the transport of one type of the photo-generated carriers [10]. The onset of the hole signal and the electron signal saturation indicate that the field extends all the way through the diode at an average field slightly higher than $20 \mathrm{~V} / \mu \mathrm{m}$. This average field is higher than that of simple $p-i-n$ diodes because additional bias is needed to deplete the buried doped layers. A uniformlyabsorbing light pulse ( $\lambda=760 \mathrm{~nm}, 0.5 \mu \mathrm{sec}$ wide) simulates energetic charged particles and the tendency of signal saturation at high bias indicates that the charge collection efficiency is not perfect but moderately sufficient.

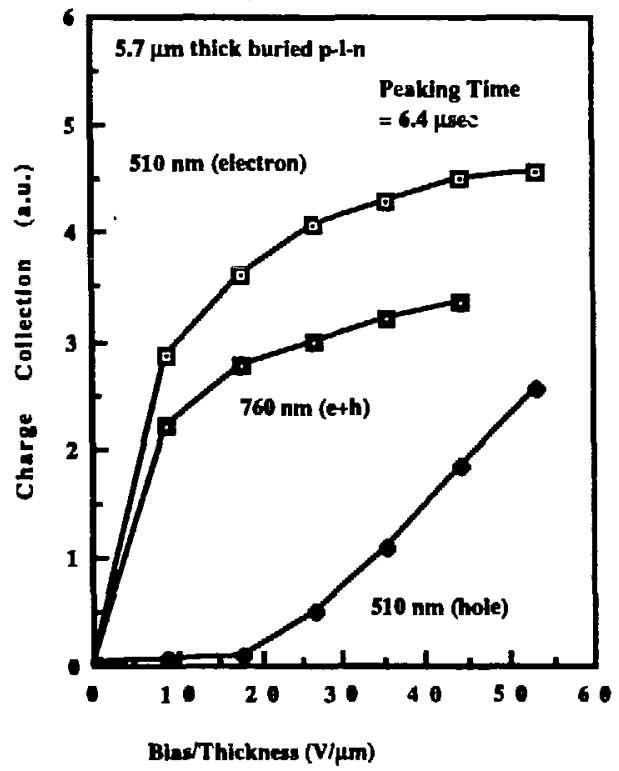

Fig.5 Pulsed light measurements on the $5.7 \mu \mathrm{m}$ thick buried p-i-n diode.

When detecting high LET particles such as $\alpha$ particles from radioisotopes with the conventional $p-i-n$ diodes, the signal size increases with bias but not with the detector thickness. The typical signal size produced by $6 \mathrm{MeV} \alpha$ particles is 20,000 electrons at the highest bias applicable for layers of different thickness. This low signal has been 
attributed to carrier recombination in the plasma column created by the $\alpha$ trajectory $[2,3,11]$.

The buried p-i-n device was exposed to $5.8 \mathrm{MeV} \alpha$ particles from a Cf- 249 source in vacuum and the signal size is shown in Fig.6 as a function of reverse-bias for three different incident angles of $\alpha$ particles. The peaking time is fixed at $6.4 \mu \mathrm{sec}$ for this measurement. For normal incidence, the collecied charge exceeds 60,000 electrons at a bias of $300 \mathrm{~V}$. The signal size at $200 \mathrm{~V}$ is about 20,000 electrons and agrees with the results of the conventional $5 \mu \mathrm{m} p$-i-n devices. The ratio of the collected charge for oblique incidence to that for the normal incidence is larger than the factor $1 / \cos \theta$, where $\theta$ is the incidence angle. This fact leads one to believe the existence of carrier recombination loss [11]. This ratio decreases with average field higher than $35 \mathrm{~V} / \mu \mathrm{m}$ and approaches the expected value of the path increase factor $(1 / \cos \theta)$. This is the expected behavior when the recombination loss becomes smaller with higher field strength. Its observation has become possible with the high electric field achieved by this buried p-i-n structure. To measure how fast carriers are collected following the $\alpha$ particle passage, the signal size was recorded with different peaking times $(0.2,0.4$, $0.8,1.6,3.2,6.4 \mu \mathrm{sec})$. Results are plotted in Fig.7. Charge collection is improved only slightly by choosing longer ptaking time.

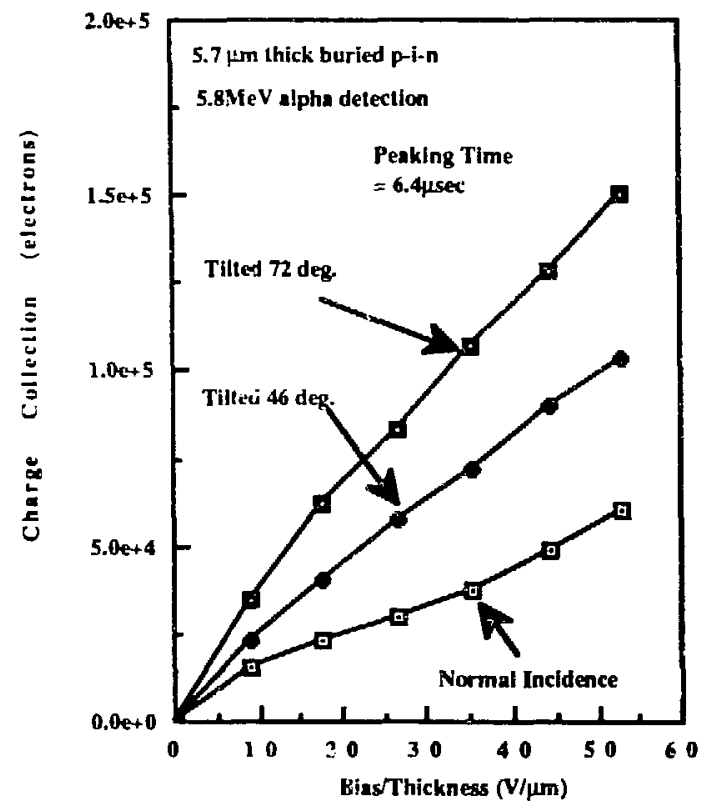

Fig.6 5.8 MeV $\alpha$ particle detection with the $5.7 \mu \mathrm{m}$ buried p$i-n$ diode described in the text. Incident angle of the $\alpha$ particles are set to $0^{\circ}, 46^{\circ}$, and $72^{\circ}$ with respect to the diode plane normal.

\section{ANALYSIS OF $\alpha$ PARTICLE DETECTION}

We attempt to develop a simple model which takes into account both "plasma erosion" and carrier recombination following an $\alpha$ particle transit through a fully-depleted a-Si:H

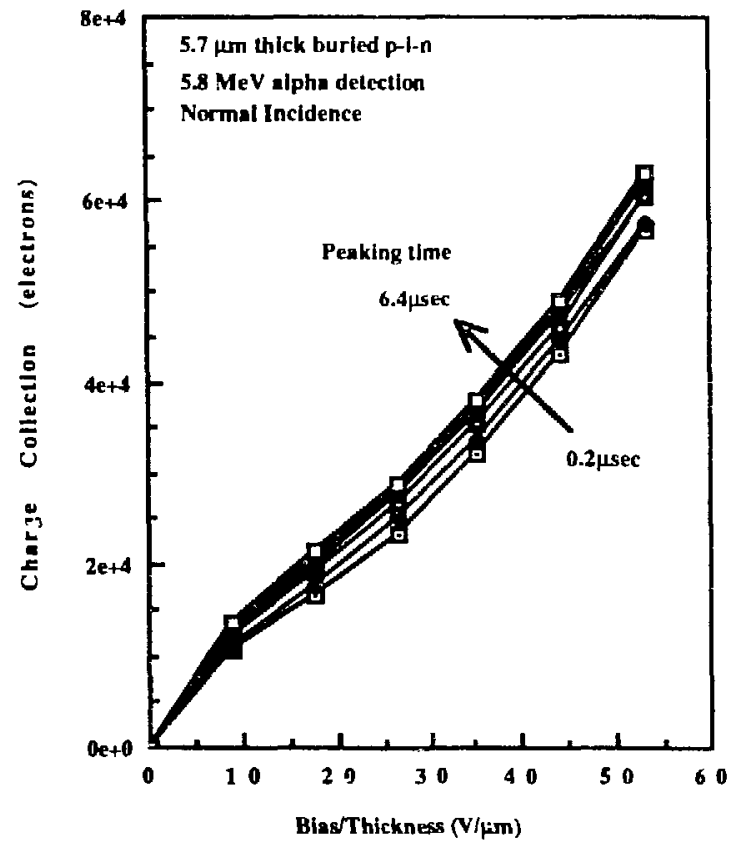

Fig.7 5.8 $\mathrm{MeV} \alpha$ particle detection with the $5.7 \mu \mathrm{m}$ buried pi-n diode described in the text. The signal size for different peaking times $(0.2,0.4,0.8,1.6,3.2,6.4 \mu \mathrm{sec})$ are shown. The charge collection is enhanced only slightly by choosing longer peaking time compared to shorter peaking time.

diode. Since Fig.7 shows no significant sign of the ballistic deficit, carrier loss during the transport is assumed to be neglegible.

The plasma erosion process in crystalline silicon has been modeled by many authors who analyzed the fission fragment detection by surface-barrier type silicon detectors and more recently by groups working on the bit-inversion error caused by energetic particle passage in digital intcgrated circuit chips. The model by Shur [12] assumes that the plasma column around the particle track is eroded by charge sweep-out at the column surface where the carrier density is at the level of the substrate doping and hence the external field is at its normal level. Carriers have to move to the surface by anbipolar diffusion since the external field is shielded inside the plasma column. Another source of the erosion current as modeled by Seibt et al. [13] comes from the space charge limited current (SCLC) from the end of the plasma column initially located at the end of the track or the end of the detector layer whichever is shorter. The column length becomes shortened with time by the SCLC. In both cases, the time derivative of the lincar carrier density $\mathbf{N}$ is proportional to the time. Since the diffusion is slow in a-Si:H, the SCLC is the dominant contribulion to the plasma erosion.

Second, we consider carrier densily change due to the volume recombination inside the plasma column. Following the treatment by Ad!er et at. [14], the time derivative of the volumetric carrier density $n$ is proportional to $n^{2}$. Converting $\mathrm{n}$ into $\mathrm{N}$ requires a detailed know!edge of the radial carricr 
distribution. Instead, we simply assume an uniform carrier distribution inside the column radius $\mathbf{r}_{\mathrm{s}}$.

When both effects are taken into account, the line carrier density is given by the solution of the special case of the socalled Riccati differential equation, which can be solved numcrically. In addition to the uniform radial carrier distribution, the external electric field is assumed uniform throughout the detector for simplicity. The column radius $\mathbf{r}_{\mathbf{s}}$ is the only filting parameter used and $r_{s}=2.3 \mu \mathrm{m}$ gives a good fit to the normal incidence case in Fig.6. The carrier density vanishes at approximatly $80 \mathrm{~ns}, 35 \mathrm{~ns}$ and $15 \mathrm{~ns}$ for field strengths of $1 \times 10^{5}, 2 \times 10^{5}, 5 \times 10^{5} \mathrm{~V} / \mathrm{cm}$, respectively. These plasma times are consistent with Fig. 7 which shows no significant ballistic deficit with peaking times as short as 200 ns. But the use of a more realistic value for the column radius $r_{\mathrm{s}}$ such as $0.1 \mu \mathrm{m}$ overestimates the recombination loss. $0.1 \mu \mathrm{m}$ is the maximum range of the most energetic delta electrons created by the $6 \mathrm{MeV} \alpha$ particle passage. This may be explained by the presence of the "latent period" during which carriers are too hot to recombine with each other as discussed in [15] and/or the assumption of the uniform carrier distribution inside the plasma may be too crude.

\section{CONCLUSION}

A novel structure of a-Si: $\mathrm{H}$ radiation detectors is fabricated by inserting two lightly-doped thin layers into the conventional $p^{+}-i-n^{+}$diode. This $p^{+}-i-p-i-n-i-n^{+}$structure decreases the electric field at the metal contacts and retards the onset of the micro-plasma breakdown thus permitting the use of higher electric fields. Consequently, the charge collection efficiency from $5.8 \mathrm{MeV} \alpha$ particles is improved by a factor of three compared to the conventional $\mathrm{p}^{+}-\mathrm{i}-\mathrm{n}^{+}$diodes. This buried $\mathrm{p}-\mathrm{i}-\mathrm{n}$ structure should also be beneficial in the detection of lower LET particles due to the faster response expected by the higher field in the bulk of the $i$ layer.

The capability for tailoring the field by inserting doped layers or a-Si alloys with different band gaps opens a way to some interesting devices. For example, controlled avalanche multiplication may become possible in the central intrinsic layer when it is sandwiched by heavily-doped layers and separated away from the metal surfaces to avoid the microplasma breakdown. Thicker a-Si:H layers may become fullydepleted by adding thin $p$ layers into the intrinsic layer which reverse the field gradient in these added layers[16]. Superlattice avalanche structures that take advantage of the band discontinuity at hetero-junctions [17] becomes promising wher the properties of a-Si based alloys such as a-SiC:H and a-SiGe: $\mathrm{H}$ are improved. These future devices will take advantage of the unique feature of the a-Si:H preparation technique, namely, the capability of making very abrupt changes in material composition.

\section{ACKNOWLEDGEMENTS}

We would like to express our appreciation to C.C. Tsai of Xerox PARC and J. Xi of Glasstech Solar, Inc. for making the samples.

\section{REFERENCES}

(1) V. Perez-Mendez, G. Cho, I. Fujieda, S.N. Kaplan, S. Qureshi and R.A. Street, presented at Mater. Res. Soc. Spring Meeting, San Diego, CA., April 24-29,1989.

[2] B. Equer and A. Karar, Nucl. Instr. Meth. A271(1988)574.

[3] J. DuBeau, T. Pochet, A. Karar, L.A. Hamel, B. Equer, J.P. Martin, S.C. Gujrathi and A. Yelon, Proc. Mater. Res. Soc. Vol.118(1988) 439.

[4] I. Fujieda, G. Cho, M. Conti, J. Drewery, S.N. Kaplan, V. Perez-Mendez, S. Qureshi and R.A. Street, LBL-27457, July,1989, presented at Int. Conf. on Amorphous Semiconductor Technology, Asheville, NC., Aug. 24-25,1989.

[5] M. Stutzmann, D.K. Biegelsen and R.A. Street, Physical Review B 35(1987)5666.

[6] F. Goulding and D. Landis, IEEE Trans. Nucl. Sci. NS29(1982)1125.

[7] R.A. Street, Physical Review B 27(1983)4924.

[8] S. Qureshi, V. Perez-Mendez, S.N. Kaplan, I. Fujieda G. Cho and R.A. Street, presented at Mater. Res. Soc. Spring Meeting, San Diego, CA., April 24-29,1989 and also at Int. Conf. on Amorphous and Liquid Semiconductors, Asheville, NC., Aug. 21-25,1989.

[9] J. DuBeau, T. Pochet L.A. Hamel B. Equer and A. Karar, presented at Mater. Res. Soc. Spring Meeting, San Diego, CA., April 24-29,1989 and also at Int. Conf. on Amorphous Semiconductor Technology, Asheville, NC., Aug. 24-25,1989.

[10] I. Fujieda, G. Cho, S.N. Kaplan, V. Perez-Mendez, S. Qureshi, W. Ward and R.A. Street, Proc. Mater. Res. Soc. Vol.118(1988)467.

[11] V. Perez-Mendez, S.N. Kaplan, W. Ward, S. Qureshi and R.A. Street, Nucl. Instr. Meth. A260(1987)195.

[12] M. Shur, IEEE Trans. Nucl. Sci. NS-33(1986)1140.

[13] W. Seibt, K.E. Sundstrom and P.A. Tove, Nucl. Instr. Meth. 113 (1973)317.

[14] D. Adler, M. Silver, A. Madan and W. Czubatyj, J. Appl. Phys. 51 (1981)6429.

[15] T.R. Oldham, J. Appl. Phys. 57(1985)2695.

[16] V. Perez-Mendez, S.N. Kaplan, G. Cho, I. Fujieda, S. Qureshi, W. Ward and R.A. Street, Nucl. Instr. Meth. A273(1988) 127.

[17] S. Jwo, M. Wu, Y. Fang, J. Hong and C. Chang, IEEE Trans. Electron Devices, ED-35(1988) 1279. 


\section{DISCLAIMER}

This doument was prepared as an account of work sponsored by the United States Government. Neither the United States Government nor any agency thereof. nor The Regents of the University of California, nor any of their employees, makes any warranty, express or implied. or assumes any legal liability or responsibility for the accuracy, completeness, or usefulness of any information, apparatus. product, or process disclosed, or represents that its use would not infringe privately owned rights. Reference herein to any specific commercial products process, or service by its trade name. trademark, manufacturer. or otherwise. does not necessarily constitute or imoly its endorsement. recommendation. or favoring by the United States Government or any agency thereof. or The Regents of the University of California. The views and opinions of authors expressed herein do not necessarily state or reflect those of the United States Government or any agency thereof or The Regents of the University of California and shall not be used for advertising or product endorsement purposes.

Lawrence Berkeley Laboratory is an equal opportunity employer 